\title{
Differentiation of Dermal Muscle in Chin Region of Prenatal Goat (Capra hircus)
}

\author{
Prabhakar Kumar $^{1 *}$, Ajay Prakash ${ }^{2}$, M.M. Farooqui ${ }^{2}$, S.P. Singh ${ }^{2}$ and Varsha Gupta ${ }^{2}$ \\ ${ }^{1}$ Department of Veterinary Anatomy, College of Veterinary \& Animal Sciences, SVPUAT, \\ Meerut - 250110 (UP), India \\ ${ }^{2}$ Department of Veterinary Anatomy, College of Veterinary Sciences \& AH, DUVASU, \\ Mathura-281 001 (UP), India \\ *Corresponding author
}

\section{Keywords}

Histology, Dermis, Skeletal Muscle, Goat, Skin, Prenatal etc.

Article Info

Accepted:

07 February 2019

Available Online:

10 March 2019

\section{A B S T R A C T}

The study was conducted on the skin of 36 goat foetus between 23 to 150 days of gestation with objective to record the sequential histological changes that take place during the differentiation of muscles in the dermis of chin region of prenatal goat. The material was divided into three groups viz. Group I (below 50 days of gestation), Group II (51-100 days of gestation) and Group III (101 days of gestation to full term) containing 12 foetii each group. The skin samples were collected and processed and stained as per standard histological technique. In the dermis of chin region the muscles began to appear as myocyte at the age of 70 days gestation and were usually localized in the deeper half of dermis. They assumed fiber like profile from 75 days gestation and from 99 days gestation the differentiating muscle fibers showed resemblance to the characters of skeletal muscles. Distinct cross striations in the muscles of dermis became evident from 106 days of gestation. From 108 days onwards the skeletal muscles gradually became pronounced and extended superficially in the dermis. These muscles were is close apposition to sinus hair follicles present in the region but were not associated to the ordinary hair follicles. The arrector pili muscles which are generally found with hair follicles of other regions were not observed in the dermis of chin region.

\section{Introduction}

The goats (Capra hircus) belong to familae Bovidae are reared throughout the world for providing the animal protein in the form of meat and milk to the human beings, and slaughter by-products, the skin and fibers as raw material for the leather and textile industries. Skin is the largest and heaviest organ of the body and makes about $16 \%$ of its weight (Hejazi et al., 2013) and consisted of epidermis, dermis and skin appendages (Saleemm et al., 2016). The appendages of skin include hair follicle, sebaceous gland, sweat gland, and arrector pili muscle. The appendages of skin that are found in dermis perform specialized physiological functions (Wildelitz - Randall et al., 1997). Arrector pili 
muscle is generally associated with hair follicles and mediates thermoregulation by contracting to increase air-trapping (Torkamani et al., 2014). In addition to presence of arrector arrector pili muscles in dermis some some study about the occurrence of skeletal muscles in dermis of lips, muzzle and eyelids has been reported (Chandra, 1966 in buffalo; Yus and Simón, 2000 in human). Eurell and Frappier (2006) in domestic animals mentioned that the skeletal muscles are associated with large sinus hairs of facial region. Although, the arrector pili muscle and skeletal muscles are normal constituent of dermis, very little attention has been put towards the differentiation of these muscle in goat. Looking into above facts the present study was planned reveal the sequential histological changes that occur during the differentiation of muscles in dermis of chin region of prenatal goat.

\section{Materials and Methods}

The study was conducted on the skin collected from chin region of thirty six apparently healthy goat embryo/ foetuses of none descript breed between 23 to 150 days of gestation. The approximate age of the embryos (upto 30 days of gestation) was estimated on the basis of foetal measures and phenotypic characteristics given by Njaa (2012) and Anonymous (2008) in goat. The age of the remaining embryos/ foetuses was estimated by using the formula derived by Singh et al (1979) in goat after interpolation of formula of Hugget and Widdas (1951). The embryos/ foetuses were divided into three age groups each with twelve embryos/ foetuses viz. group-I (0-50 days), group-II (51-100 days) and group-III (101-150 days). The skin samples were processed by routine paraffin embedding technique. The skin tissues were sectioned $(5-6 \mu)$ and stained with hematoxylin and eosin for general architecture, Weigert's resorcin fuchsin stain for elastic fibres, Mallory's triple stain
(Crossman, 1937) and Masson's trichrome stain for collagen fibres (Luna, 1968).

\section{Results and Discussion}

In the dermal tissue of goat foetues the myocytes began to appear at the age of 70 days gestation in chin region and were usually localized in the deeper half of dermis and their long axes were generally oriented perpendicular to the epidermal surface. The myocytes were elongated in appearance and had fiber like profile; these had darkly stained flattened or oval nuclei with eosinophilic cytoplasm. Several other myocytes showed varying stages of differentiation and usually had cylindrical cone like filmentous eosinophilic extensions at their one end. Many other myocytes had such extensions on their both ends. Several of these extensions showed rounded ends where as many other had tapering ends. At 75 days gestation these were relatively more elongated in appearance and had fiber like profile. These had darkly stained flattened or oval nuclei with eosinophilic cytoplasm (Fig. 1). Appearance of these muscle cells with similar cytological characters in the dermis of sheep foetus was reported by Hejazi et al (2013) at 8 week of gestation. In 99 and 100 days old goat foetuses the differentiating muscle fibers showed resemblance to the characters of skeletal muscles. In 102 days old goat foetus thin skeletal muscles oriented in different planes were present in patches in the deeper part of dermis of chin region. Although these muscles had indistinct cross striations but their nuclei were located peripherally (Fig. 2). The beginning of appearance of cross striations indicated the formation of actin and myosin protein of myofilaments to form the alternate dark and light bands. Similar views were expressed by Allen (1978) in vertebrate who mentioned that myofibrillar proteins were synthesized and grouped into interacting thick and thin filaments that formed transverse 
striations in skeletal muscles. The presence of skeletal muscles running in all directions in dermis of lips, muzzle and eyelids has been reported by Chandra (1966) in buffalo. Yus and Simón (2000) mentioned that in human striated muscle fibers are a normal constituent of the dermis of the eyebrow; eyelid and nose; and lips and chin. Eurell and Frappier (2006) in domestic animals mentioned that the skeletal muscles are associated with large sinus hairs of facial region. However, in present study the skeletal muscles and sinus hair follicles were observed in the dermis of chin region only. At 106 and 108 days of gestation the occurrence of skeletal muscles in deeper part of dermis in chin region was more pronounced as these muscles were relatively longer and were extending upward towards the superficial part of dermis at various angles.

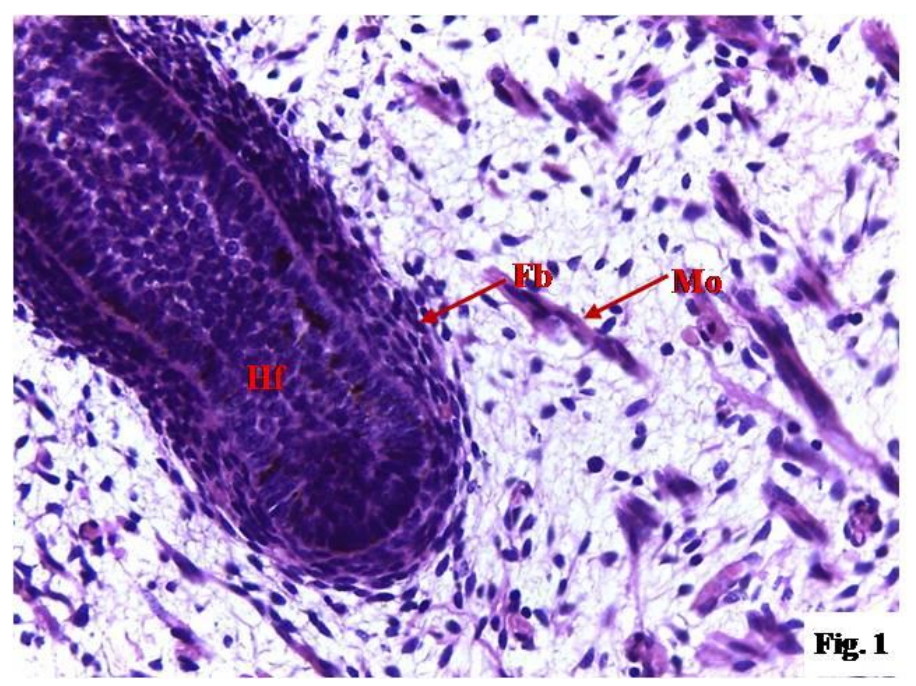

Fig.1 Photomicrograph of skin of chin region of 75 day old goat foetus showing developing hair follicles ( $\mathrm{Hf})$, fibroblast $(\mathrm{Fb})$, and myocyte (Mo) dermis.

Haematoxylin and Eosin, X 400

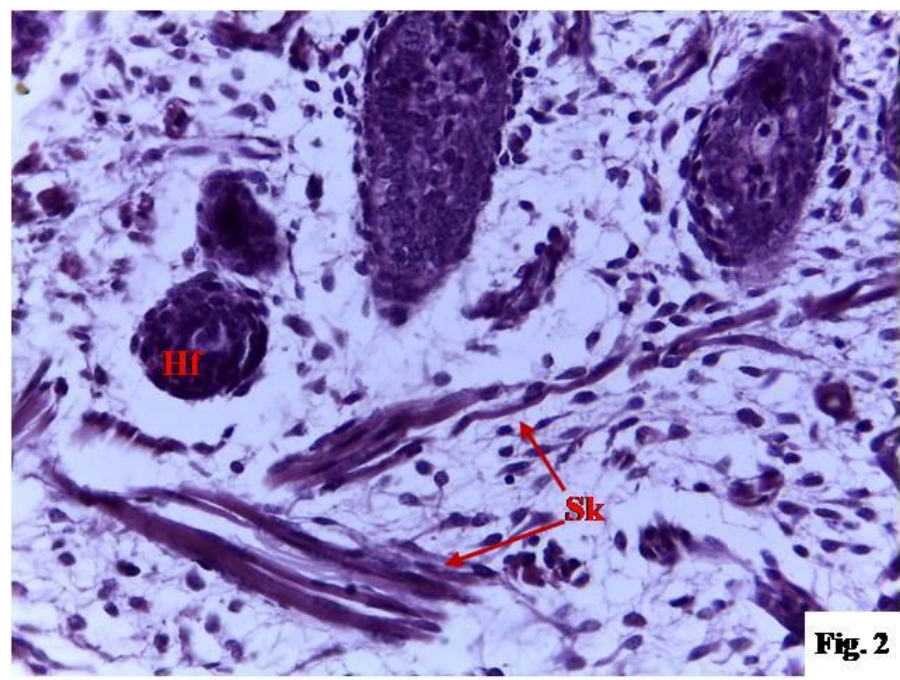

Fig.2 Photomicrograph of skin of chin region of 102 day old goat foetus showing patches of developing skeletal muscles (Sk) in dermis and hair follicles (Hf).

Haematoxylin and Eosin, X 400 


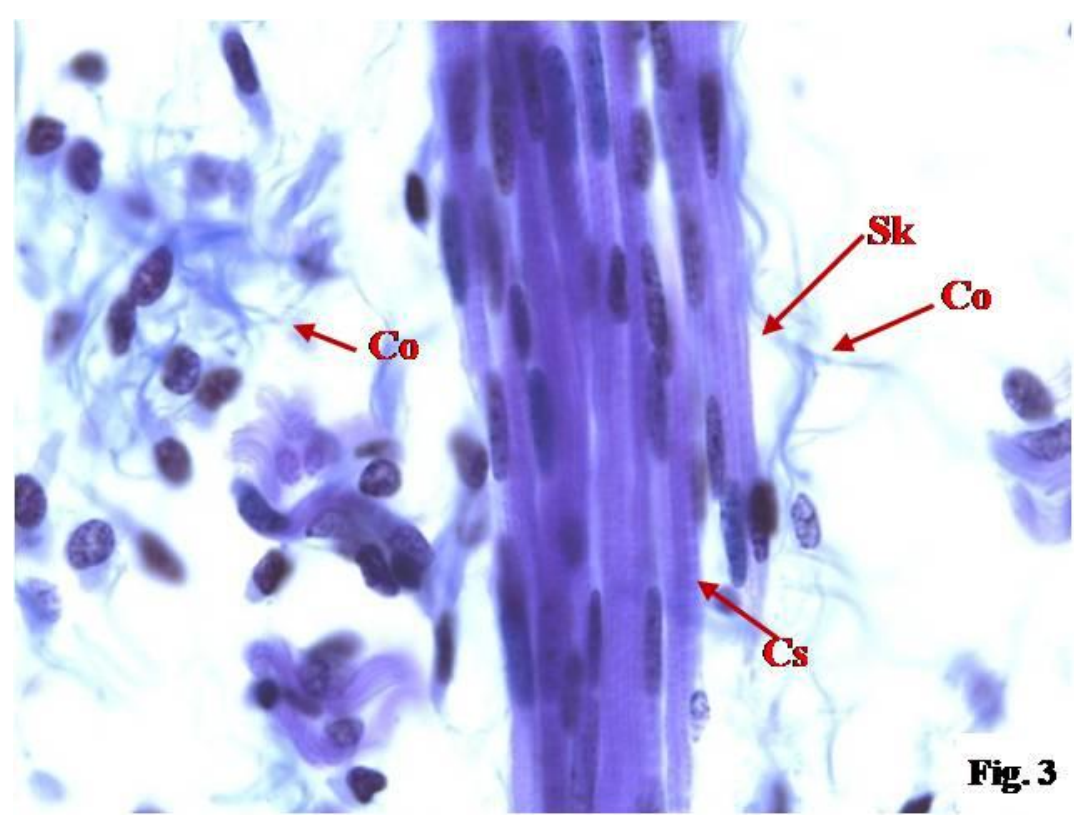

Fig.3 Photomicrograph of skin of chin region of 108 day old goat foetus showing cross striation (Cs) in skeletal muscle (Sk) and collagen fibers (Co) in dermis. Mallory's triple stain, X 1000

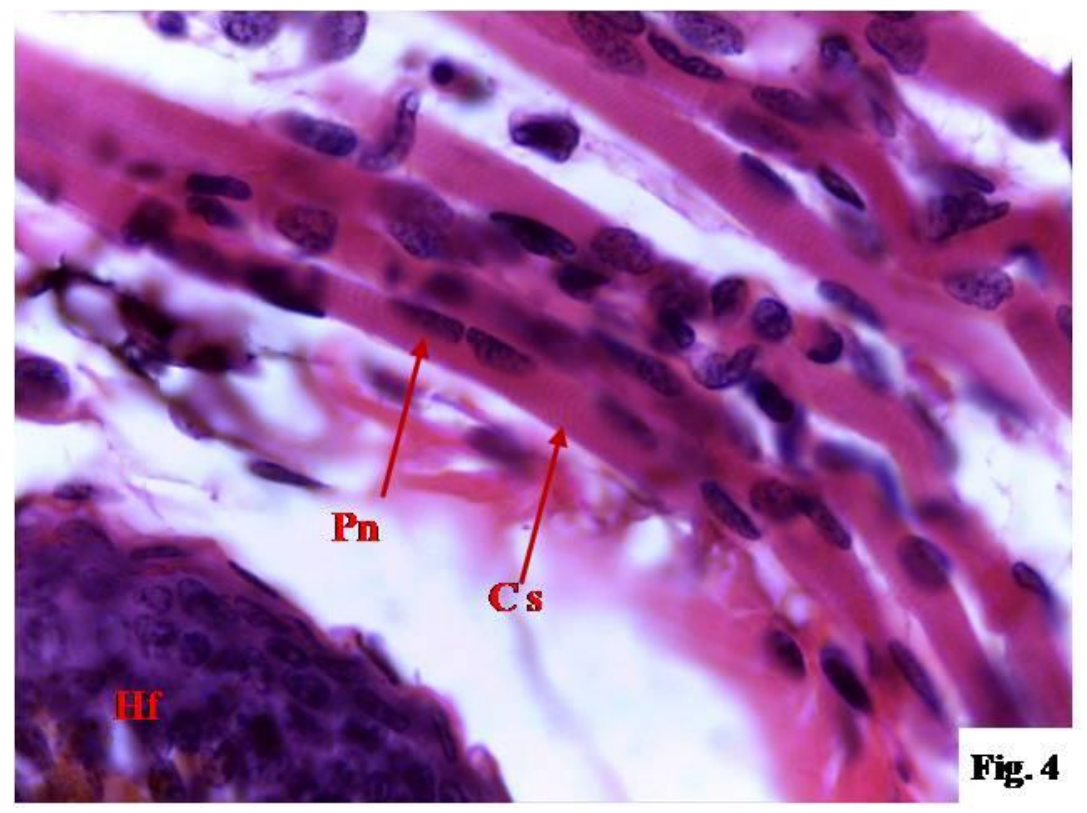

Fig.4 Photomicrograph of skin of chin region of 134 day old goat foetus showing cross striation $(\mathrm{Cs})$ and peripheral nuclei $(\mathrm{Pn})$ in skeletal muscle near hair follicle $(\mathrm{Hf})$, of dermis. Haematoxylin and Eosin, X 1000 


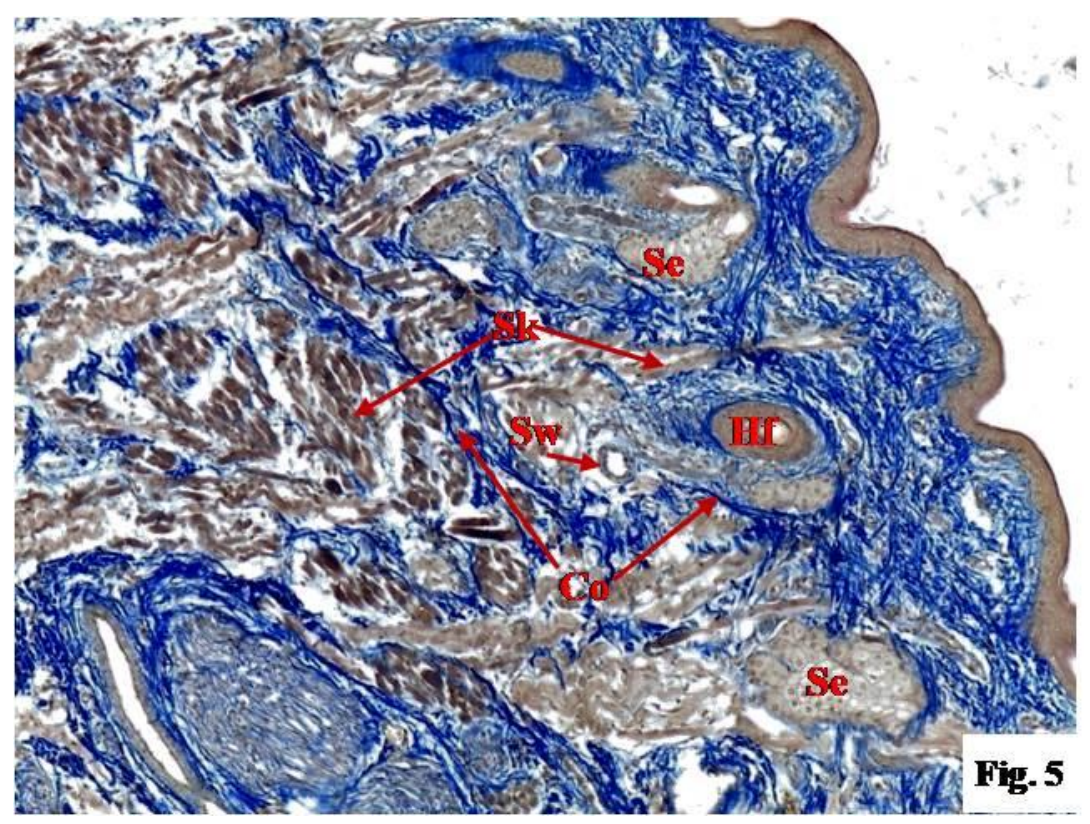

Fig.5 Photomicrograph of skin of chin region of 150 day old goat foetus showing collagen fibers (Co) and skeletal muscle (Sk), sweat gland (Sw) and sebaceous gland (Se) in dermis.b Masson's trichrome stain, X 100

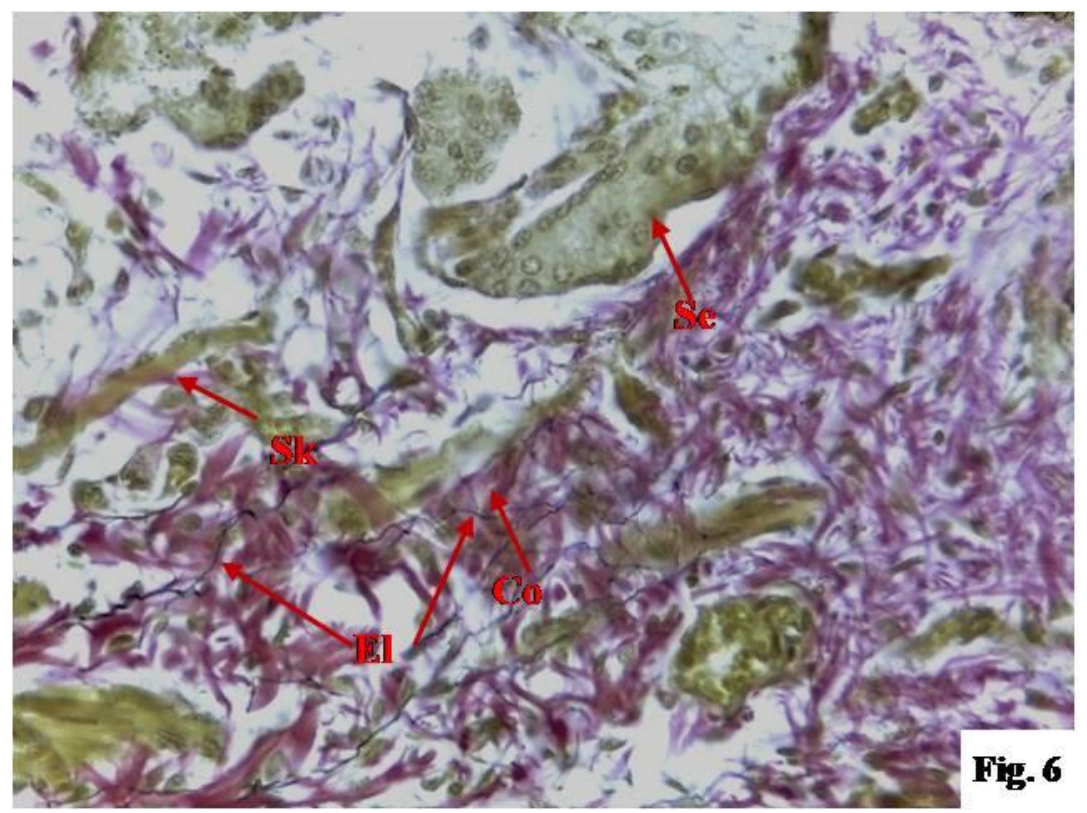

Fig.6 Photomicrograph of skin of chin region of 145 day old goat foetus showing collagen fibers $(\mathrm{Co})$, elastic fibers (El), skeletal muscle (Sk) and sebaceous gland (Se) in dermis. Weigert's resorcin fuchsin stain, X 400 


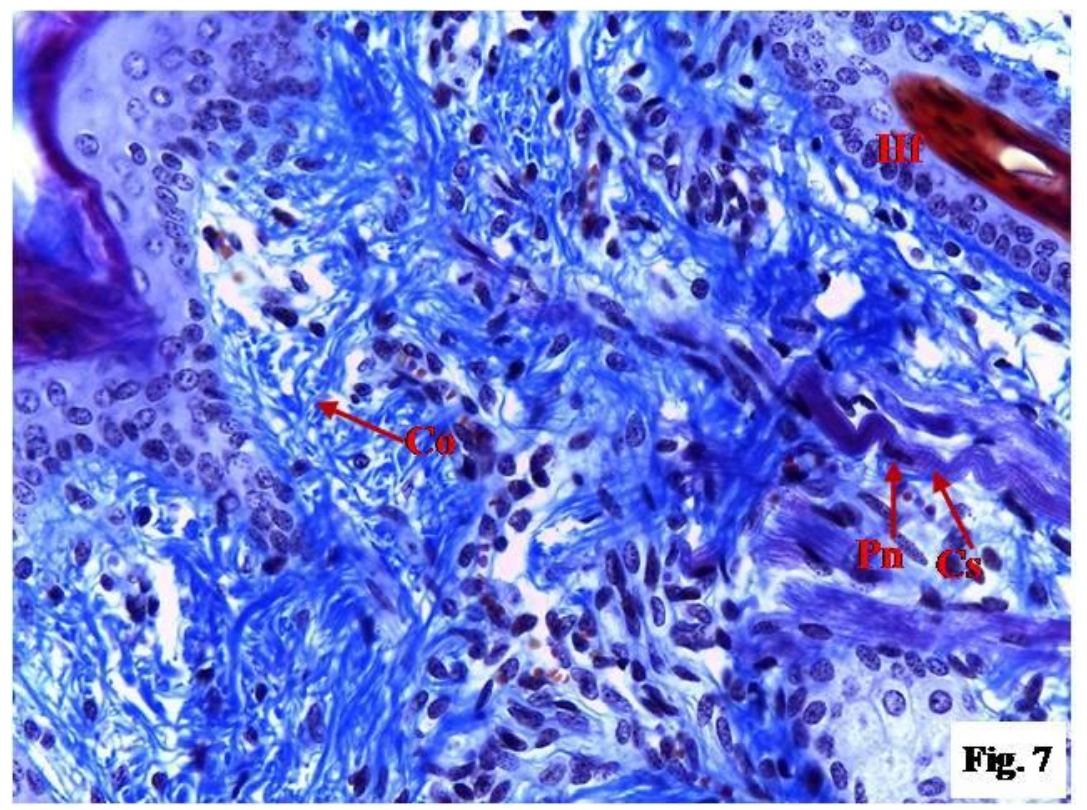

Fig.7 Photomicrograph of skin of chin region of 134 day old goat foetus showing cross striation (Cs) and peripheral nuclei (Pn) in skeletal muscle near hair follicle (Hf) in dermis. Mallory's triple stain, X 400

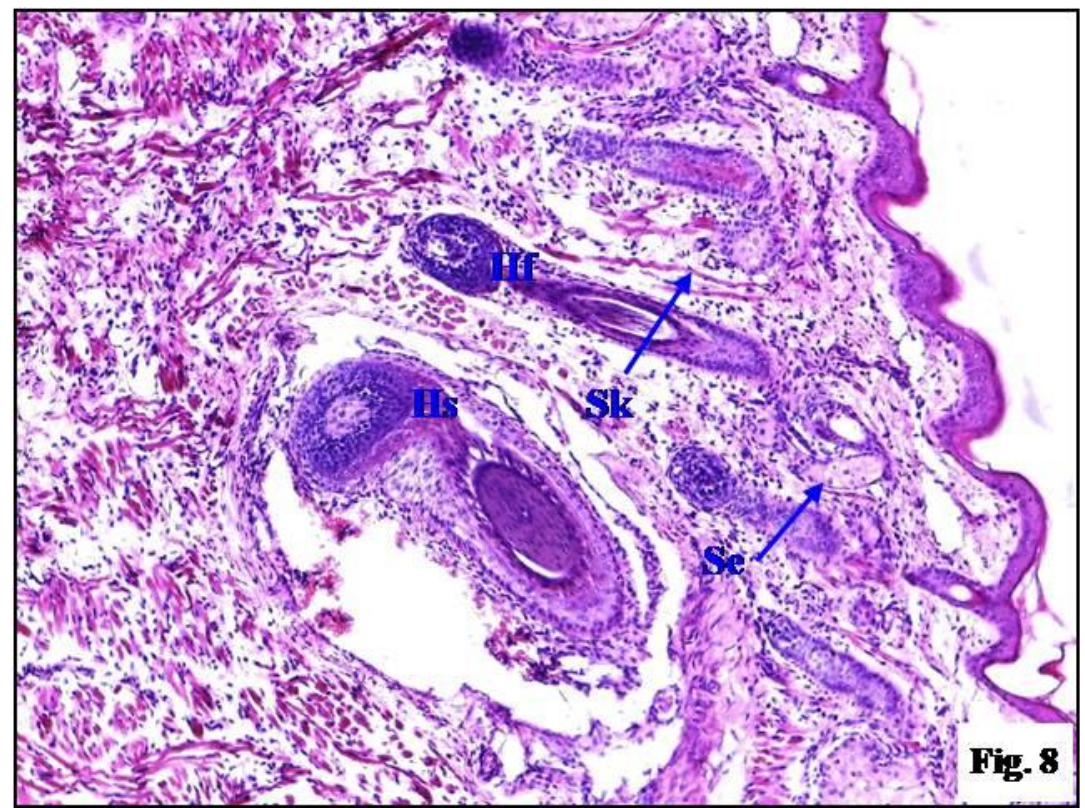

Fig.8 Photomicrograph of skin of chin region of 150 day old goat foetus showing hair follicle (Hf), sinus hair follicle (Hs), skeletal muscle (Sk), sebaceous gland (Se) in dermis.

Haematoxylin and Eosin, X 100

Moreover, the cross striations in the skeletal muscle fibers became distinct and these had multiple elongated nuclei placed at the periphery (Fig. 3). Similar cytological 
characters of skeletal muscles had been described in domestic animals by Eurell and Frappier (2006) and Hyttel et al., (2010). With the advancement of gestational age the skeletal muscle fibers extended superficially and at 121 days these extended above the terminal part of hair follicles (hair bulb) where these were observed in patches. In 131 and 134 days old foetus the dermal skeletal muscles were relatively longer as compared to the previous stages and were present throughout dermis especially near the hair follicles (Fig. 4), however, in deeper zone the skeletal muscles occupied most area where as in superficial zone collagen fibers predominated. At 145, 148 and 150 days gestation the reticular zone of dermis was chiefly formed by the bundles of sekeletal muscles separated by thin bundles of collagen fibers (Fig. 5). Patches of elastic fibers were also found among the bundles of collagen fibers in reticular part of dermis from 145 days gestation (Fig. 6). The skeletal muscles from reticular zone of dermis extended in the papillary zone where these were observed in all part of papillary zone except subepidermal area. This was in agreement with the findings of Yus and Simón (2000) in human, they observed skeletal numerous superficial muscles in chin region. In present study no arrector pili muscles were observed in chin region (Fig. 7 and 8).

In conclusion, the myocytes started appearing the in dermis of chin regions at 70 days gestation in their deeper part. These myocytes showed the characteristics of skeletal muscles from 102 days gestation. The cross striations in the dermal muscles of chin region became distinct from 106 days of gestation and from 118 days of gestation onwards the skeletal muscles extended to superficial part of dermis. There was no any arrector pili muscles associated with hair follicles of chin region of goat foetus.

\section{References}

Allen, E.R. 1978. Development of vertebrate skeletal muscle. Am. Zool. 18, 101-111.

Anonymous 2008. Stages of goat fetal development.

www.thekebun.wordpress. com/2008/09/28 / stages-of-goat-fetaldeve.

Chandra, G. 1966. Histological and certain histochemical studies on the skin of Bos bubalis. MVSc Thesis submitted to Agra University, Agra, India

Crossman, G.A. 1937. A modification of Mallory's connective tissue stain with discussion of principles involved. Anatomical Record, 69: 33-38.

Eurell, J.A. and Frappier, B.L. (2006). Dellmann's Textbook of Veterinary Histology.; 6th ed Blackwell Publishing.; Ames.; Iowa.; USA.; 2006.; 420 pp. ISBN 0-7817-4148-3.

Hejazi, S., Yaghoubi, S., Delghandi, M. and Javid, F. 2013. Histogenesis study of skin in sheep. Life Sc. Jour., 10, 194198.

Hugget, A.S.G. and Widdas, W.F. 1951. The relationship between mammalian foetal weight and conception age. J. Physio. 114, 306-317.

Hyttel, P., Sinowatz, F. and Vejlsted, M. 2010. Essentials of domestic animal embryology. Sounders Elsvier.

Luna, L.G. 1968. Manual of Histological Staining Methods of the Armed Forces Institute of pathology. $3^{\text {rd }}$ Ed., McGraw Hill, New York, USA.

Njaa, B.L. 2012. Kirkbride's Diagnosis of Abortion and Neonatal Loss in Animals. $4^{\text {th }}$ Ed., John Wiley and Sons, Ltd., USA.

Saleemm, M.A., Habeeb, R.S. and Abdullah, M.A. 2016. Comparative histological study of the skin layers of black goats in north Iraq. AL-Qadisiyah Journal of Vet. Med. Sci., 15(2), 2 25-30. 
Singh, Y., Sharma, D.N. and Dhingra, L.D. 1979. Morphogenesis of the testis in goat. Ind. J Ani. Sc., 49(11), 925-931.

Torkamani, N., Rufaut, N.W., Jones, L. and Sinclair, R.D. 2014. Beyond goosebumps: does the arrector pili muscle have a role in hair loss? Int. $J$. Trichol., 6, 88-94.

Wildelitz -Randall, B., Ting -Xin, J., Alexandra, IV., Sheree, A., Ting -
Berreth, F.Y., Han -Sung, J. and Cheng-Meng, C. 1997. Molecular Histology in Skin Appen-dage Morphogenesis. Microse. Res. Tech., $38,452-465$.

Yus, E. S. and Simón, P. 2000. Striated muscle: a normal component of the dermis and subcutis in many areas of the face. The Am. J Dermatopathol., 22(6),503-509.

\section{How to cite this article:}

Prabhakar Kumar, Ajay Prakash, M.M. Farooqui, S.P. Singh and Varsha Gupta. 2019. Differentiation of Dermal Muscle in Chin Region of Prenatal Goat (Capra hircus). Int.J.Curr.Microbiol.App.Sci. 8(03): 640-647. doi: https://doi.org/10.20546/ijcmas.2019.803.079 